\title{
A Case Report of Atypical Hemolytic Uremic Syndrome Presenting with Massive Proteinuria
}

\author{
Rabia Akhtar ${ }^{1}$, Diane Triolo ${ }^{2, *}$ \\ ${ }^{1}$ Department of Internal Medicine, St. Joseph’s Regional Medical Center, New York Medical College \\ ${ }^{2}$ Division of Nephrology, Department of Internal Medicine, St. Joseph’s Regional Medical Center \\ *Corresponding author: akhtar.rabia@hotmail.com
}

Received July 08, 2015; Revised July 20, 2015; Accepted July 28, 2015

\begin{abstract}
Atypical hemolytic uremic syndrome (HUS) is most commonly due to dysregulation of the alternative complement pathway. It is characterized by the classic triad of hemolytic anemia, thrombocytopenia, and acute kidney injury. Treatment includes supportive care including hemodialysis along with steroids, plasmapheresis, and eculizumab. We report a case of a 22 year old female who was diagnosed with atypical HUS and was also noted to have a severe degree of proteinuria, nearly 24 grams in the 24 hour urine collection. Proteinuria is observed in cases of atypical HUS because of the glomerular leakage of protein due to severe endothelial and epithelial injury. However, there is little data directly addressing the degree of proteinuria observed in these patients. This leads us to ask whether the degree of proteinuria has any prognostic significance or impact on disease progression and recovery.
\end{abstract}

Keywords: atypical hemolytic uremic syndrome, Eculizumab, proteinuria

Cite This Article: Rabia Akhtar, and Diane Triolo, "A Case Report of Atypical Hemolytic Uremic Syndrome Presenting with Massive Proteinuria.” American Journal of Medical Case Reports, vol. 3, no. 9 (2015): 278-281. doi: 10.12691/ajmcr-3-9-4.

\section{Introduction}

Hemolytic Uremic Syndrome can be divided into typical and atypical causes. Typical HUS is a diagnosis reserved for children and is associated with the appearance of Shiga toxin-producing Escherichia coli, E. coli O157:H7. Atypical HUS is a disorder distinguished by the absence of Shiga toxin-producing E. coli infection. It can be further subdivided into primary causes that are due to complement dysregulation or secondary causes including infections such as Streptococcus pneumonia, human immunodeficiency virus, influenza A; malignancy; cancer; chemotherapy and ionizing radiation; bone marrow or solid organ transplantation; pregnancy; HELLP syndrome, malignant hypertension, glomerulopathies, systemic diseases such as SLE, antiphospholipid antibody syndrome, and scleroderma [1]. Atypical hemolytic uremic syndrome is a rare condition, affecting approximately about two per one million people in the United States [1]. It is characterized by thrombocytopenia, hemolytic anemia and acute kidney injury. Thrombotic thrombocytopenic purpura (TTP) is a disease that also presents with thrombocytopenia and hemolytic anemia but usually presents with predominant CNS involvement while HUS predominantly presents with renal involvement. Initial management of aHUS includes supportive care including RBC transfusion, platelet transfusion, and dialysis. Eculizumab and plasmapharesis are also used in the definitive management of this condition.

\section{Case Presentation}

A 22 year old Caucasian female with a past medical history of dysmenorrhea, on oral contraceptive pills, initially presented to her PMD's office with complaints of epigastric abdominal pain for two days. She described the pain as constant and progressively worsening, associated with nausea and vomiting. The patient also reported noticing dark urine for several days although denied any frank blood and also denied any diarrhea. She also reported that she had upper respiratory tract symptoms including runny nose, dry cough and subjective fevers about a week prior for which she took some over-thecounter medicine for her cold symptoms. She went to her PMD's office and was noted to have scleral icterus so was instructed to come to our emergency department for further evaluation. Upon presentation, she was in fact noted to have scleral icterus and mild epigastric abdominal tenderness. Initial laboratory tests revealed hemoglobin of $10.9 \mathrm{~g} / \mathrm{dL}$; severe thrombocytopenia with platelets of 16, 000/ $\mathrm{mm}^{3}$; BUN, $61 \mathrm{mg} / \mathrm{dL}$; Creatinine, $2.58 \mathrm{mg} / \mathrm{dL}$; bicarbonate, $21 \mathrm{mEq} / \mathrm{L}$; total bilirubin, $3.0 \mathrm{mg} / \mathrm{dL}$; LDH, 1935 U/L. C3 and C4 levels were within normal limits. Direct Coomb's test was negative. Peripheral smear was reviewed and showed schistocytes, no nucleated RBCs and very few platelets. On urinalysis, she had grossly red in color urine, cloudy appearance, moderate bilirubin, specific gravity $>1.030$, large blood, $>300 \mathrm{mg} / \mathrm{dl}$ of protein, positive nitrite, small leukocyte esterase, 0-4 RBC's, 5-9 WBC's, moderate bacteria. Additionally, her 
urine pregnancy test, antinuclear antibody, and HIV antibody were all negative. On abdominal ultrasound, she was found to have echogenic kidneys bilaterally. In summary, this is a 22 year old female patient who presented with abdominal pain, scleral icterus, and hematuria and was found to have hemolytic anemia, thrombocytopenia, and acute kidney injury with proteinuria.

Our patient was admitted to the medical ICU with a suspected diagnosis of TTP/HUS. Nephrology and Hematology consultations were obtained. She was initially started on steroids, plasmapharesis as well as hemodialysis. After failing to respond to plasmapharesis and with normal ADAMTS13 levels, she was started on eculizumab therapy while more workup was performed. She had an initial 24-hour urine collection which showed nearly 24 grams of protein in a total of $1269 \mathrm{ml}$ of urine. She underwent a kidney biopsy which showed diffuse, thrombotic microangiopathy involving all glomeruli, arterioles, and focal small arteries of a severe nature; global collapsing glomerulopathy; and diffuse acute tubular injury (Figure 1). She also developed hypertension during her hospital stay and was started on multiple antihypertensive medications. She eventually improved and was discharged on hospital day number 26 with plans to continue on hemodialysis and eculizumab infusions as an outpatient. Upon discharge, her hemoglobin was $9.1 \mathrm{~g} / \mathrm{dL}$, platelets of $50,000 / \mathrm{mm}^{3}$, Creatinine of $4.47 \mathrm{mg} / \mathrm{dL}, \mathrm{LDH}$ of 629 , and on urinalysis she still had large blood, > $300 \mathrm{mg} / \mathrm{dL}$ proteinuria. Over the course of the next several months, her renal function improved as evidenced by her improving GFR and decrease in the amount of proteinuria and was eventually able to be taken off dialysis. During the most recent follow-up visit, her creatinine trended down to 1.15 mg/dL; LDH, 156 IU/L; hemoglobin, 10.9 g/dL; and more remarkably the degree of proteinuria decreased with her most recent urinary protein:creatinine ratio of $385 \mathrm{mg} / \mathrm{g}$. She is currently still following with Hematology for continued biweekly infusions of eculizumab and Nephrology for monitoring of renal function.
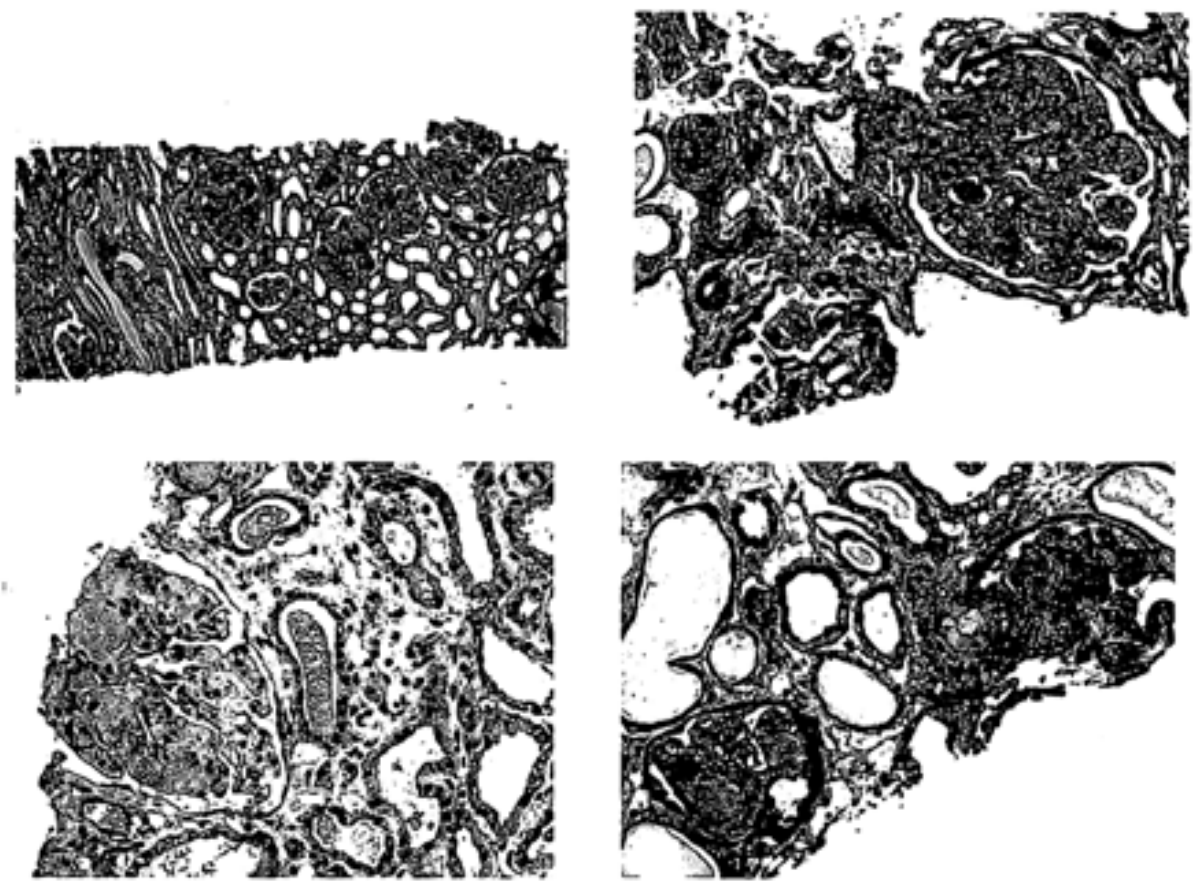
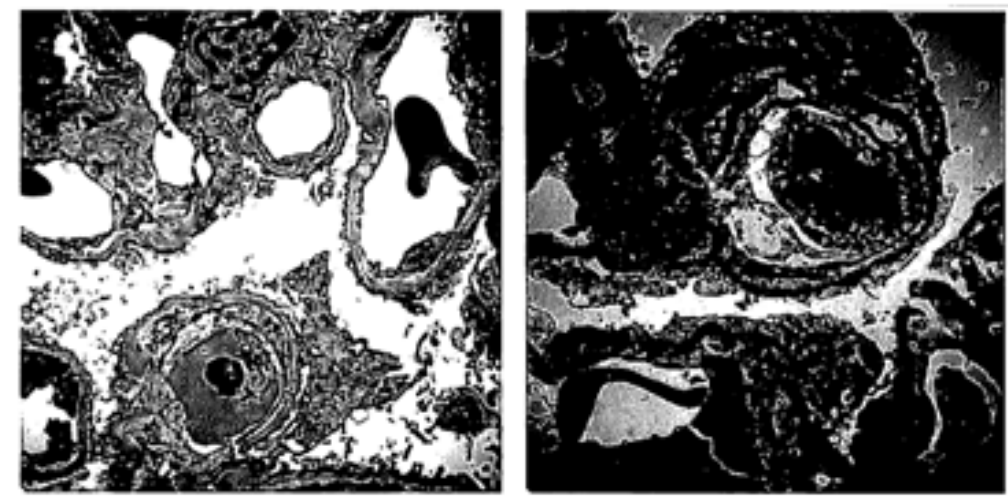

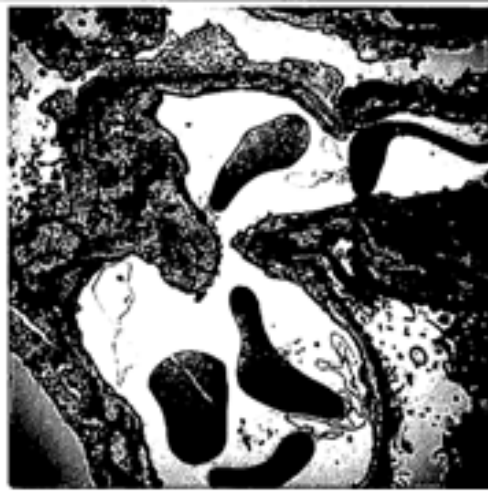

Figure 1.

\section{Discussion}

There are multiple etiologies of atypical HUS and some include primary causes such as complement dysregulation, and secondary causes such as infections like streptococcus pneumoniae and human immunodeficiency viral infection, 
drug toxicity, pregnancy or autoimmune disorders like SLE. In regards to primary HUS, it is either due to gene mutations of complement proteins or antibodies to complement factors. Mutations in the complement factor $\mathrm{H}$ gene is the most commonly identified abnormality in the complement pathway [2]. Microvascular lesions of HUS consists of vessel wall thickening with endothelial swelling and accumulation of proteins and cell debris in the subendothelial layer, creating a space between endothelial cells and the underlying basement membrane of affected microvessels [3].

TTP is an entity similar to HUS, although distinct. Both are known to cause thrombocytopenia and microangiopathic hemolysis but from different mechanisms in each disease. 5 different types of arteriolar stenosis are observed overall: 1) deficiency in ADAMTS13, a vWF cleaving protease, leads to the accumulation of unusually large $\mathrm{vWF}$ (ULVWf), platelet aggregation, and platelet thrombi; 2) platelet fibrin thrombosis like in patients with DIC, 3) tumor cell invasion of the microvasculature in patient with metastatic cancers; 4) microvascular vasculitis; 5)thrombotic angiopathy as seen in HUS [4]. There is overlap between TTP and HUS in that partial ADAMTS13 deficiency may occur in patients with complement-mediated HUS [5]. In a number of cases it is not possible to distinguish between TTP and HUS because there may be normal ADAMTS13 levels in TTP, or no defects identified in the complement pathway in HUS [5].

The initial management of HUS includes supportive care such as RBC and platelet transfusions when necessary, stopping nephrotoxic drugs that may have triggered HUS, initiation of dialysis in cases for worsening renal failure that is refractory to medical therapy. Definitive management is with plasmapharesis, Eculizumab, a monoclonal antibody to C5, and if necessary eventual renal transplant. Plasmapharesis has been thought to be effective due to its ability to remove mutated proteins and antibodies to complement factor $\mathrm{H}$ and restore the patient's blood with normal complement proteins and is most effective when initiated within the first 24 hours [6]. Eculizumab works by binding to C5, prohibiting its cleavage, and subsequently preventing the production of terminal complement proteins [6]. Eculizumab is also used as preventive therapy in those patients with increased risk of recurrence [6]. There are variable outcomes in patients undergoing renal transplant depending on the complement pathway mutation involved. Unfortunately there are a significant proportion of patients affected with recurrence of HUS following renal transplantation.

There are several widely accepted prognostic indicators in TTP/HUS. Some poor prognostic indicators carrying high mortality include duration of illness before onset of HUS $>14$ days, anuria $>3$ days, age $<18$ months and $>5$ years, non-diarrhea associated HUS, HUS associated with convulsion and hypertension, WBC > 30, 000/ $\mathrm{mm}^{3}$, platelets $<30,000 / \mathrm{mm}^{3}$, creatinine level $>700 \mu \mathrm{mol} / \mathrm{L}$ and serum potassium level 5.6 to $7.5 \mathrm{mmol} / \mathrm{L}$ [7]. A unique finding in our patient is the degree of proteinuria she exhibited. Upon initial presentation, she exhibited nearly 24 grams of protein in the 24 hour urine collection. The degree of proteinuria decreased to 385 milligrams at subsequent follow-up visits nearly 1 year later. We question whether proteinuria has any significance on the disease progression, chance of recovery, or risk of recurrence in adults with atypical hemolytic uremic syndrome.

The exact mechanism as to why proteinuria occurs in hemolytic uremic syndrome is not clearly understood. Some explanations are that it is due to a complementmediated injury to the glomerular filtration barrier [8], podocyte dysfunction via mutations in diacylglycerol kinase $\varepsilon$ (DGKE) [9], and disruption of vascular endothelial growth factor function [10]. Nevertheless, proteinuria is a parameter that has been studied as a function of recovery in children affected with hemolytic uremic syndrome. In fact, one way renal recovery has been assessed in children with TTP/HUS is by monitoring the incidence of proteinuria, hypertension, and edema at follow-up. One study found that measurement of the protein/creatinine ratio in an early-morning sample of urine is a simple, cost-effective, and noninvasive means of monitoring the progress of patients with diarrheaassociated hemolytic-uremic syndrome [11]. In one metaanalysis in which the long-term renal prognosis of diarrhea-associated hemolytic uremic syndrome was reviewed, some underlying factors studied were proteinuria, hypertension and GFR [12]. The pooled estimate of renal sequelae was $25 \%$, hypertension was $10 \%$, and proteinuria was $15 \%$ [12]. There was no stratification of patients depending on the degree of proteinuria among individual studies. Another study found that screening for microalbuminuria, within the first 6-18 months following an episode of post-diarrheal HUS, increased the sensitivity for predicting later sequelae from 22 to $66.7 \%$, compared to screening for overt proteinuria alone [13].

Upon our review of this topic, we found little in the current literature directly addressing proteinuria or the clinical implications associated with it in adult patients afflicted with atypical hemolytic uremic syndrome. The current data that is available looks at proteinuria specifically in children with HUS, in patients with post diarrheal HUS, or simply the incidence of proteinuria, but does not emphasize on the quantification or prognostic significance of the degree of proteinuria specifically in patients with atypical hemolytic uremic syndrome.

\section{Conclusion}

After reviewing the current literature available, some questions we still have are whether the degree of proteinuria has any significance on the long-term renal outcome of patients with atypical hemolytic uremic syndrome. For example, if we further stratify patients depending on the degree of proteinuria will we find that those with more severe proteinuria carry a poorer outcome and less chance for renal recovery. By identifying these patients we can perhaps treat them more aggressively at the onset of the diagnosis and prevent long term sequelae.

\section{Funding}

None. 


\section{Conflict of Interest}

None.

\section{References}

[1] Loirat C, Frémeaux-Bacchi V. Atypical hemolytic uremic syndrome. Orphanet Journal of Rare Diseases 2011; 6:60.

[2] Barbour T, Johnson S, Cohney S, et al. Thrombotic microangiopathy and associated renal disorders. Nephrol Dial Transplant 2012; 27(7):2673-85.

[3] Noris M, Remuzzi G. Disease of the Month: Hemolytic Uremic Syndrome. J Am Soc Nephrol 2005; 16(4):1035-1050.

[4] Tsai HM. Thrombotic thrombocytopenic purpura and the atypical hemolytic uremic syndrome: an update. Hematol Oncol Clin North Am 2013; 27:565-584.

[5] Feng S, Eyler S, Zhang Y, et al. Partial ADAMTS13 deficiency in atypical hemolytic uremic syndrome. Blood 2013; 122(8):14871493.
[6] Waters AM, Licht C. aHUS caused by complement dysregulation: new therapies on the horizon. Pediatric Nephrology 2011; 26(1):41-57.

[7] Malla K, Mallat T, Hanif M. Prognostic Indicators in Haemolytic Uraemic Syndrome. Kathmandu Univ Med J 2004; 2(4):291-296.

[8] Menon M, Chuang P, He CJ. The Glomerular Filtration Barrier: Components and Crosstalk. International Journal of Nephrology 2012.

[9] Noris M, Mele C, Remuzzi G. Podocyte dysfunction in atypical haemolytic uraemic syndrome. Nat Rev Nephrol 2015.

[10] Eremina V, Jefferson JA, Kowalewska J, et al. VEGF Inhibition and Renal Thrombotic Microangiopathy. The New England Journal of Medicine 2008; 358(11):1129-1136.

[11] Milford DV, White RH, Taylor CM. Prognostic significance of proteinuria one year after onset of diarrhea-associated hemolyticuremic syndrome. J Pediatr 1991; 118(2):191-4.

[12] Garg AX, Suri RS, Barrowman N, et al. Long-term Renal Prognosis of Diarrhea-Associated Hemolytic Uremic Syndrome: A Systematic Review, Meta-analysis, and Meta-regression. JAMA. 2003; 290(10):1360-1370.

[13] Lou-Meda R, Oakes RS, Gilstrap JN, et al. Prognostic significance of microalbuminuria in postdiarrheal hemolytic uremic syndrome. Pediatr Nephrol 2007; 22(1):117-120. 